\title{
Carbon Dioxide Laser-assisted Giant Sialolith Removal from Wharton's Duct
}

\author{
${ }^{1}$ Baskaran Muthiah, ${ }^{2}$ Varun Muthuraman
}

\begin{abstract}
Carbon dioxide laser is a highly effective tool for numerous intraoral surgical procedures. Its use for sialolithotomy has been well documented in the literature because of its ability to achieve hemostasis at the time of incision of the Wharton's duct and the painless nature of the procedure requiring just topical anesthesia or local anesthetic nerve block at the most. As carbon dioxide laser has a high absorption coefficient for water, saliva in the oral cavity acts as a substrate and hence the deeper penetration of the beam and subsequent injury to adjacent structures is prevented. We describe a case of a giant sialolith of the Wharton's duct, which was successfully removed by carbon dioxide laser-assisted sialolithotomy.
\end{abstract}

Keywords: Absorption coefficient, Carbon dioxide laser, Giant sialolith.

How to cite this article: Muthiah B, Muthuraman V. Carbon Dioxide Laser-assisted Giant Sialolith Removal from Wharton's Duct. Int J Laser Dent 2015;5(1):26-28.

\section{Source of support: Nil}

\section{Conflict of interest: None}

\section{INTRODUCTION}

Carbon dioxide laser was granted safety clearance from US Food and Drug Administration (FDA) in 1976 for its use in soft tissue surgery. The wavelength of carbon dioxide lasers $(10600 \mathrm{~nm})$ falls in the infrared range thereby rendering it invisible. A quartz fiber incorporating 630-nm coaxial helium-neon laser is used as an aiming beam. Sialolithasis most commonly occur in the Wharton's duct ${ }^{1}$ because its secretion being rich in mucous content and torturous nature of the duct leading to stasis of secretion in the duct causing mineralization that results in sialolith formation. Giant sialoliths are uncommon; but when they do occur, they are associated with suppuration of the submandibular salivary gland and also localized inflammation of the duct and surrounding tissues making the tissues friable

\footnotetext{
${ }^{1}$ Professor and Head, ${ }^{2}$ Postgraduate Trainee

${ }^{1,2}$ Department of Oral and Maxillofacial Surgery, Rajas Dental College, Tirunelveli, Tamil Nadu, India

Corresponding Author: Varun Muthuraman, Postgraduate Trainee, Department of Oral and Maxillofacial Surgery Rajas Dental College, Tirunelveli, Tamil Nadu, India, Phone: +919841017997, e-mail: drmvarun@gmail.com
}

and hyperemic. Accordingly, the carbon dioxide lasers that can effectively cut and coagulate the tissues are best suited when compared with conventional scalpel incision. The purpose of this article is to describe a case of sialolithotomy with carbon dioxide laser and substantiate its effectiveness.

\section{CASE REPORT}

A 27-year-old male patient reported to our Department of Oral and Maxillofacial Surgery with a chief complaint of severe pain over the right side of the lower jaw and bad taste in the mouth since 2 weeks. Clinical examination revealed an acutely tender swelling over the right submandibular region and swelling intraorally in the floor of the mouth. The odontogenic causes were ruled out. Palpation of the floor of the mouth revealed a hard mass along the line of the Wharton's duct. On further investigation, the Orthopantomography (OPG) (Fig. 1) showed a large radio opaque mass superimposed in the mandibular second and third molar region. A diagnosis of submandibular sialolithiasis was established. A computed tomography (CT) scan was performed to exclude the possibility of infection involving other facial spaces in the neck due to sialadenitis. The CT scan (Fig. 2) showed a calcified mass of $2.0 \times 1.5 \mathrm{~cm}$ in the floor of the mouth. The use of carbon dioxide laser was advantageous as the tissues were inflamed and friable and scalpel incision would have caused more bleeding, which would have further necessitated general anesthesia. A lingual nerve block

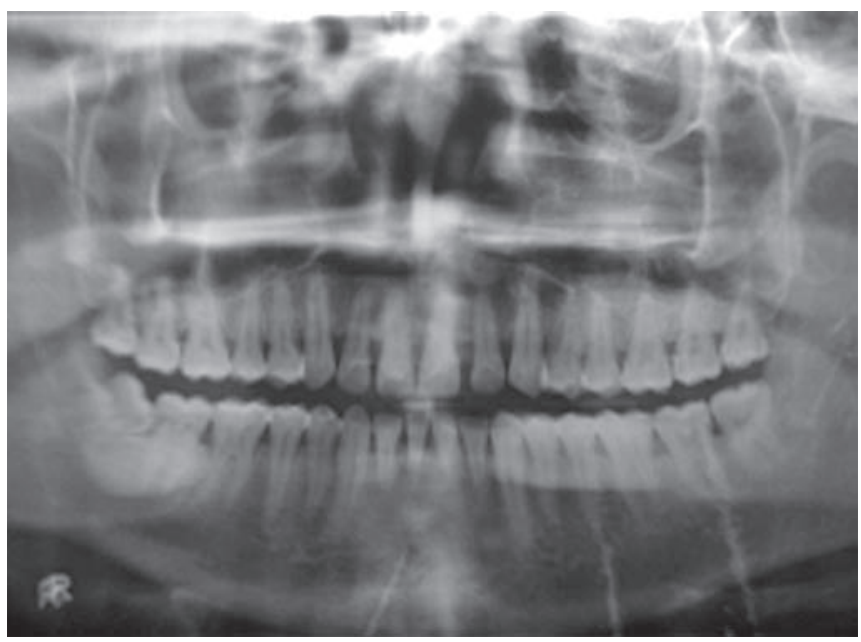

Fig. 1: Orthopantomography showing sialolith superimposed in the region of second molar 
and infiltration anesthesia were administered. A carbon dioxide sealed off type laser (model name-CAPTAIN 30) manufactured from Seoul, South Korea (Fig. 3) was used. Its wavelength is $10.6 \mu$ and a power setting of $6.5 \mathrm{~W}$ in pulsed mode was used to make an incision over the mucosa of the floor of the mouth over the sialolith (Fig. 4). No bleeding was observed at this point of time. A giant

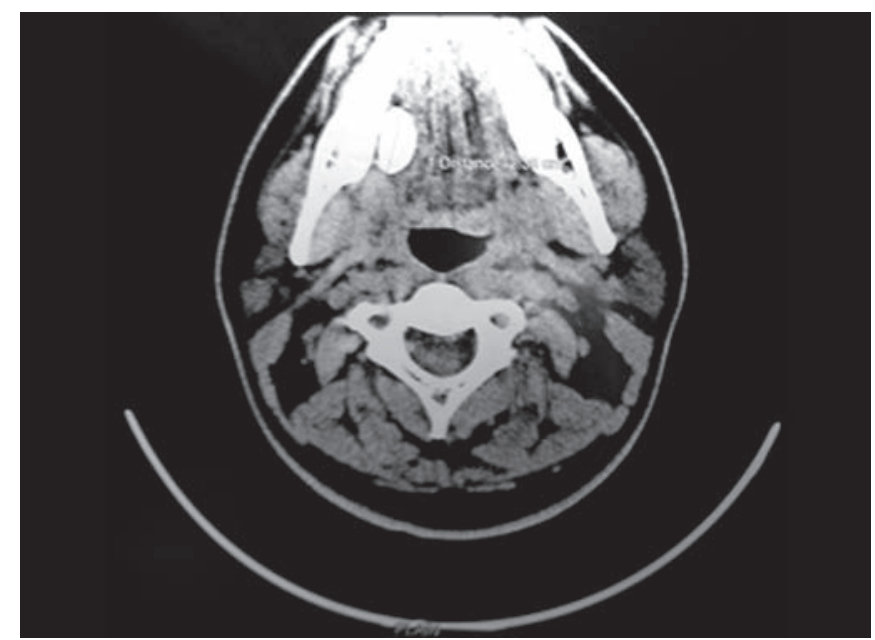

Fig. 2: Computed tomography scan showing calcified mass in the floor of the mouth

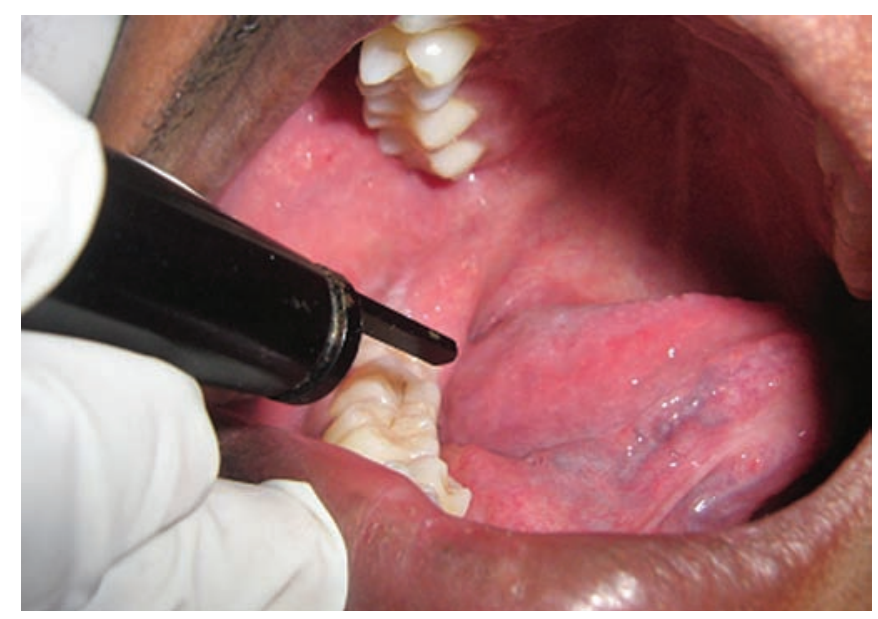

Fig. 4: Carbon dioxide laser-assisted sialolithotomy

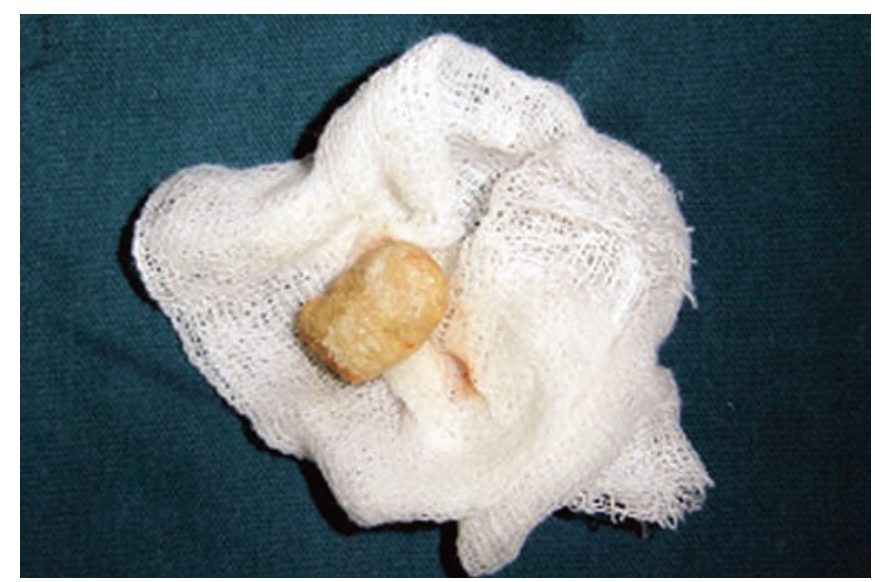

Fig. 6: Delivered sialolith sialolith was exposed and after slight dissection with a hemostat (Fig. 5), the sialolith was picked up and delivered in total (Fig. 6). The gland was bimanually kneaded to drain the pus from the Wharton's duct. This maneuver is important to prevent the recurrence of infection in the gland. A single suture was placed over the anterior region in the floor of the mouth (Fig. 7) and the duct was not

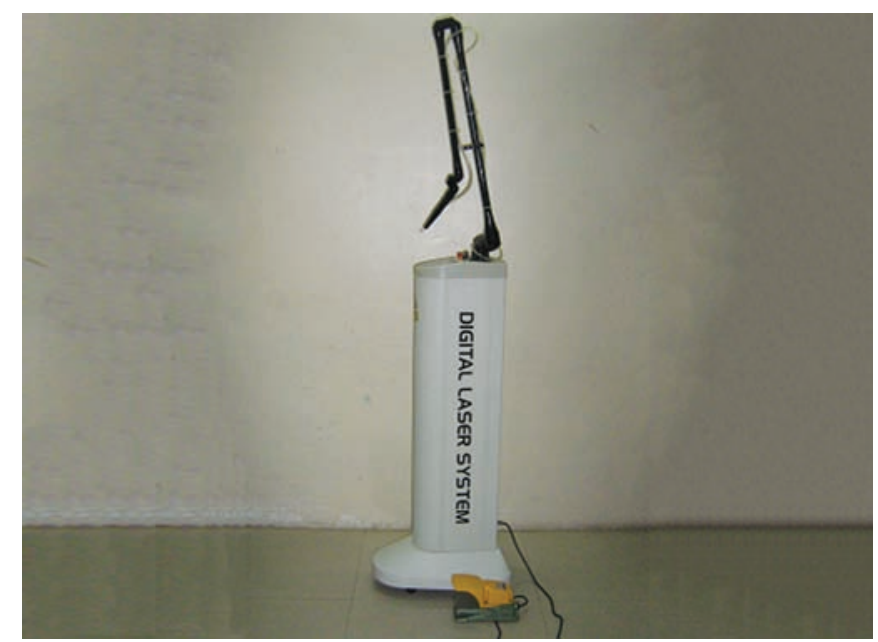

Fig. 3: Carbon dioxide laser (CAPTAIN 30, South Korea)

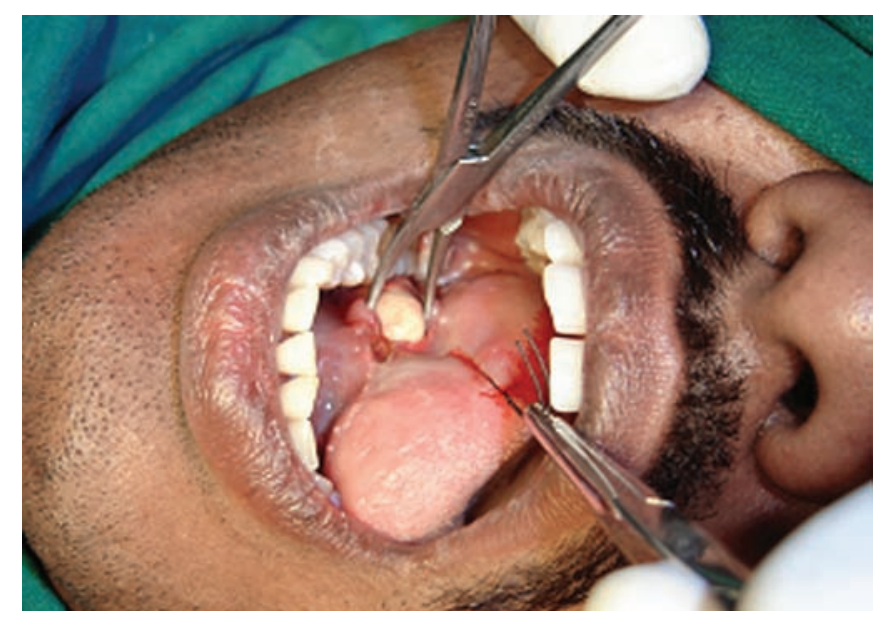

Fig. 5: Dissection with hemostat

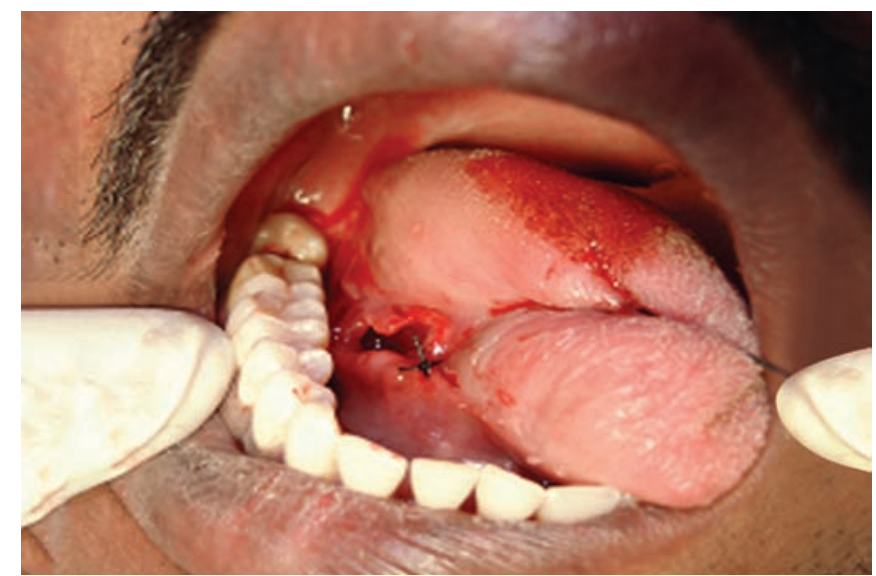

Fig. 7: Suture over anterior region only 
sutured to prevent stenosis and left to heal by secondary intension. On 2 weeks postoperative review the incision in the floor of the mouth had healed along with resolution of the submandibular swelling. On further follow up, there was no recurrence of the submandibular gland swelling and its function was normal.

\section{DISCUSSION}

The techniques for sialolithotomy were described by Combes et $\mathrm{al}^{2}{ }^{2}$ Baurmash, ${ }^{3}$ and Zenk et al. ${ }^{4}$ All of these techniques involve the identification of the lingual nerve, because it crosses over the submandibular duct at the region of the mandibular second molar. In our case, this was not required because the size of the sialolith was large enough that it could be palpated to be present just under the mucosa. Hence, when incising over the mucosa with carbon dioxide laser, the sialolith not only prevents laser radiation to reach the lingual nerve, but also prevents injury to the inferior Wharton's duct lining.

The inherent advantages in using carbon dioxide laser are its excellent healing, pain control, and decreased collateral tissue damage that minimizes the chance of duct stenosis when compared with the use of cautery or a diode laser. Topical anesthesia will usually suffice this procedure, but local anesthesia was administered in this case because of associated sialadenitis and sialectasis.
Patient with anxiety of scalpel can also be adequately managed by laser excision. Pain control is excellent and the technique is minimally invasive. Complications reported in the literature are ranula that is due to injury to the sublingual gland and other nerve injuries; but these usually occur with a more proximally located sialolith.

Hence, it can be concluded that sialolithotomy assisted by carbon dioxide laser is a pain-free and minimally invasive procedure ${ }^{5}$ and is one that has been adequately supported in the literature.

\section{REFERENCES}

1. Lustmann J, Regey E, Melamed Y. Sialolithiasis: a survey on 245 patients and a review of the literature. Int J Oral Maxillofac Surg 1990 Jun;19(3):135-138.

2. Combes J, Karavidas K, Mcgurk M. Intraoral removal of proximal submandibular stones-an alternative to sialoadenectomy? Int J Oral Maxillofac Surg 2009 Aug;38(8):813-816.

3. Baurmash HD. Submandibular salivary stones: current management modalities. J Oral Maxillofac Surg 2004 Mar;62(3): 369-378.

4. Zenk J, Constantinidis J, Al-Kadah B, Iro H. Transoral removal of submandibular stones. Arch Otolaryngol Head Neck Surg 2001 Apr;127(4):432-436.

5. Yang SW, Chen TA. Transoral carbon dioxide laser sialolithectomy with topical anaesthesia. A simple, effective, and minimally invasive method. Int J Oral Maxillofac Surg 2011 Feb;40(2):169-172. 